\title{
Identification of sialylated glycoproteins from metabolically oligosaccharide engineered pancreatic cells
}

\author{
Yuan Tian', Ruben T Almaraz², Caitlin H Choi ${ }^{1}$, Qing Kay Li ${ }^{1}$, Christopher Saeui ${ }^{2}$, Danni Li ${ }^{1}$, Punit Shah', \\ Rahul Bhattacharya ${ }^{2}$, Kevin J Yarema ${ }^{2}$ and Hui Zhang ${ }^{1 *}$
}

\begin{abstract}
In this study, we investigated the use of metabolic oligosaccharide engineering and bio-orthogonal ligation reactions combined with lectin microarray and mass spectrometry to analyze sialoglycoproteins in the SW1990 human pancreatic cancer line. Specifically, cells were treated with the azido $\mathrm{N}$-acetylmannosamine analog, 1,3,4-BuzManNAz, to label sialoglycoproteins with azide-modified sialic acids. The metabolically labeled sialoglyproteins were then biotinylated via the Staudinger ligation, and sialoglycopeptides containing azido-sialic acid glycans were immobilized to a solid support. The peptides linked to metabolically labeled sialylated glycans were then released from sialoglycopeptides and analyzed by mass spectrometry; in parallel, the glycans from azido-sialoglycoproteins were characterized by lectin microarrays. This method identified 75 unique N-glycosite-containing peptides from 55 different metabolically labeled sialoglycoproteins of which 42 were previously linked to cancer in the literature. A comparison of two of these glycoproteins, LAMP1 and ORP150, in histological tumor samples showed overexpression of these proteins in the cancerous tissue demonstrating that our approach constitutes a viable strategy to identify and discover sialoglycoproteins associated with cancer, which can serve as biomarkers for cancer diagnosis or targets for therapy.
\end{abstract}

Keywords: Sialylated glycoproteins, Metabolic oligosaccharide engineering, Pancreatic cancer cells

\section{Introduction}

Altered patterns of glycosylation are a universal feature of cancer with sialic acids - which are a group of unusual amino-modified acidic sugars ubiquitously displayed on the outer ends of mammalian glycan chains [1] - playing an especially prominent role as tumorassociated carbohydrate antigens (TACAs) [2,3]. TACAs, in general, are involved in many biological and pathological processes, such as regulating cellular and molecular interactions by either masking recognition sites or serving as recognition determinants [4]. More specifically, altered sialylation of tumor cell surfaces is associated with several critical malignant properties that include invasiveness and metastatic potential [5-7]. Cell surface sialylation is controlled by several factors, which

\footnotetext{
* Correspondence: hzhang32@jhmi.edu

'Department of Pathology, Johns Hopkins University, 400 N. Broadway, Room 4011, Baltimore MD 21287, USA

Full list of author information is available at the end of the article
}

include regulation of metabolic flux of ManNAc into the sialic biosynthetic pathway $[8,9]$, changes in the expression and enzymatic activities of sialyltransferases and sialidases, and the availability of candidate penultimate glycan termini on glycoproteins that serve as acceptor sites for sialylation $[5,10]$.

An interesting observation made about two decades ago was that cancer cells expressed the non-human "Neu5Gc" form of sialic acid at much higher levels than normal tissue [11]. The accumulation of Neu5Gc in tumors was attributed to scavenge of dietary Neu5Gc [12], which was ultimately biosynthetically incorporated into sialylated TACAs at much higher levels than into the sialoglycans of healthy cells. Building on the observation, the enhanced ability of tumors to over-express types of exogenouslysupplied sialic acid other than the canonical human "Neu5Ac" form, has been proposed as a new approach to cancer therapy with Neu5Prop and Neu5Phenyl analogs being evaluated for immunotherapy purposes 
[13]. Similarly, sialic acid precursors with chemical functional groups such as the ketone [14] or azide [15] not normally found in mammalian sugars have been applied to the preferential delivery of diagnostic or therapeutic agents to tumors. The emerging success of these strategies is evident in the recent in vivo demonstration that azidomodified sialic acids derived from corresponding ManNAc metabolic precursors are highly expressed in tumors compared to healthy tissues [16].

While a variety of high-throughput approaches have been used to study sialylated glycoproteins [17], which include lectin affinity $[6,10,18]$, titanium dioxide affinity chromatography [19], strong cation exchange columns [20], conditional hydrazide chemistry $[7,18,21-24]$, or the combination of diagonal chromatographic technology and neuraminidase treatments [25], the above-mentioned metabolic oligosaccharide engineering strategy is an emerging chemical method to analyze glycoproteins that has become increasingly used in the past decade $[15,26]$. In this approach, an unnatural monosaccharide with a bioorthogonal functional group can be introduced into the biosynthetic pathways of living cells and incorporated into cellular glycoproteins. The bio-orthogonal functional group can function as a chemical handle and be tagged with complementary reactive partners via covalent ligation reactions. An important feature of metabolic oligosaccharide engineering is that the labeling of glycans can occur in both living cells and animals and the method can be used to manipulate and study glycosylation events [27]. Accordingly, this method is becoming valuable for glycoproteomics studies in both eukaryotic and bacterial systems with investigation of cancer a particularly active area of research [27-32].

Based on the selective in vivo incorporation of exogenously supplied monosaccharides into tumors, as discussed above, we reasoned that a metabolic oligosaccharide engineering approach could be used to meet a present need in understanding cancer, which is to more thoroughly characterize the role of sialoglycoproteins in tumor progression. As a caveat, the selective incorporation of nonnatural sialic acids into tumors could simply be a consequence of the increased metabolic activity of cancer cells compared to most normal cells found in the body. However, a previous study where we treated the human SW1990 pancreatic cancer cell line with the "high flux" $1,3,4-\mathrm{O}-\mathrm{Bu}_{3} \mathrm{ManNAc}$ analog that increases levels of natural sialic acid, showed that increased sialylation did not occur evenly across all glycoproteins. Instead, certain glycoproteins did not experience a measurable increase in sialylation while important cancer-related markers including CD44 and integrin $\alpha 6$ [9] as well as EGFR [33] experienced increased levels of sialic acid of 2-fold or more. Increased levels of natural sialic acid, however, hold less potential to manipulate or study the system compared to a similar approach where non-natural sialosides are metabolically installed into cancer-associated glycans. Therefore, in the present study, we investigated whether treatment of SW1990 cells with the non-natural azidomodified sialic acid precursor 1,3,4-O- $\mathrm{Bu}_{3} \mathrm{ManNAz}$ [34] would similarly preferentially label cancer-associated sialoglycans and, by doing so, provide a method for the discovery, isolation, and study of this important class of tumor-promoting molecules.

To experimentally verify the incorporation of azido-sialic acids into cancer-associated glycans, we treated SW1990 cells with 1,3,4-O-Bu $\mathrm{Bu}_{3} \mathrm{ManNAz}$ and adapted methods previously used in our groups (as outlined in Figure 1) to extract and enrich azido-labeled glycopeptides and glycoproteins based on the modified Staudinger reaction first reported by the Bertozzi group [15]. Once enriched, the samples were analyzed by mass spectrometry to determine the identity of proteins labeled by azido-modified glycans and by lectin analysis to gain a sense of the composition of the actual glycan structures. Once this knowledge was in hand, we analyzed clinical samples to verify that proteins that were identified were selectively associated with cancer compared to healthy tissue.

\section{Results}

\section{Incorporation of azido-groups into cellular} glycoconjugates

Metabolic oligosaccharide engineering-based incorporation of 1,3,4-O- $\mathrm{Bu}_{3} \mathrm{ManNAz}$ into the glycoconjugates of various cell lines including SW1990 was recently investigated [34], suggesting that $50 \mu \mathrm{M}$ of analog was an adequate concentration to robustly label sialoglycans without inducing any noticeable cytotoxicity, growth inhibition, or other "off-target" effects. The series of experiments shown in Figure 2 confirmed that $50 \mu \mathrm{M}$ was an appropriate ana$\log$ concentration to conduct the metabolic labeling reported in the current study. In particular, SW1990 cells were treated with $50 \mu \mathrm{M} 1,3,4-\mathrm{O}-\mathrm{Bu}_{3} \mathrm{ManNAz}$ and then labeled by adding Click-iT reaction cocktail to conjugate fluorescent dyes to the azide groups of sialic acids. Fluorescent microscopy showed strong azide-derived signal in 1,3,4-O-Bu ${ }_{3} \mathrm{ManNAz}$ treated cells and virtually no background signal in the untreated controls (Figure 2A). In complementary experiments designed to verify the metabolic incorporation of azido analogs into the glycoconjugates of SW1990 cells, proteins were extracted from treated or untreated cell lysates, biotinylated via a modification of the Staudinger ligation reaction [15], separated by electrophoresis, and evaluated using HRP-streptavidin (Figure 2B). The data showed the detection of numerous biotinylated proteins of a broad ranges of molecular weights in lysates derived from 1,3,4-O- $\mathrm{Bu}_{3} \mathrm{ManNAz}$ treated cells but virtually no signal from lysates labeled in a similar manner but derived from the untreated 


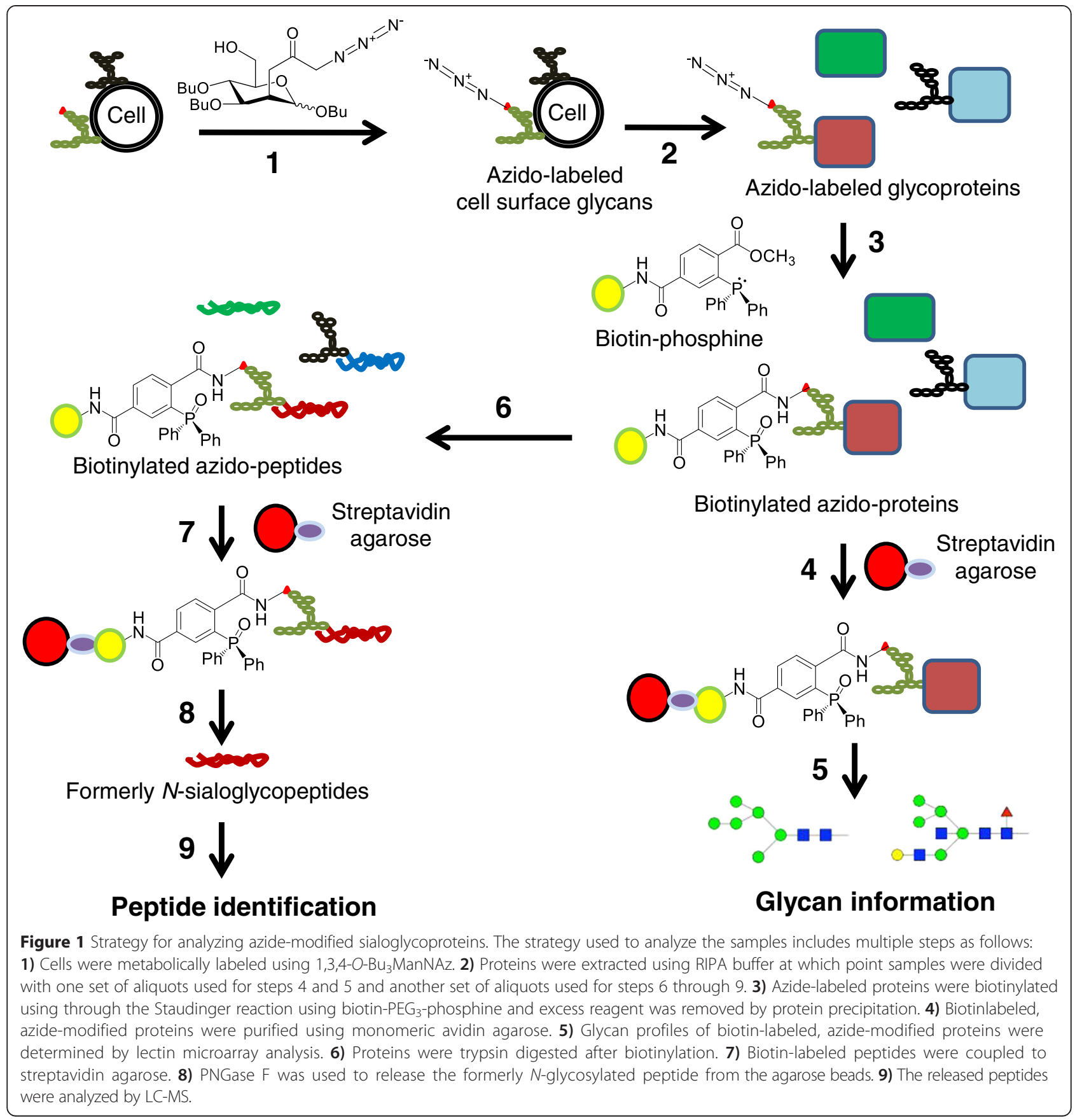

control cells. Silver staining (Figure 2C) showed that similar amounts of proteins were loaded for each blot, confirming that the biotin tag was specifically added only to azido-sugars present in the glycans of analog treated cells via the intended bioorthogonal ligation reaction between the azide and phosphine groups.

\section{Lectin microarray analysis}

To investigate the glycosylation patterns on azido-sialic acid containing glycoproteins in pancreatic cancer cells, our previously developed lectin microarray [35] was used to bind biotinylated glycoproteins (Figure 1, Steps 4 and 5) that had first been affinity purified by monomeric avidin agarose resin from 1,3,4-O- $\mathrm{Bu}_{3} \mathrm{ManNAz}$-treated and control cells (Figure 1, Steps 1-3). Streptavidinconjugated Dylight 549 was used to detect the biotinylated azido-sialoglycoproteins on lectin microarray. No detectable binding signal above background was obtained from samples from the control cells without ManNAz analog treatment (Figure 3). In contrast, glycoproteins obtained from treated cells showed high binding intensity to 10 of the 38 lectins on the array with 
(A)

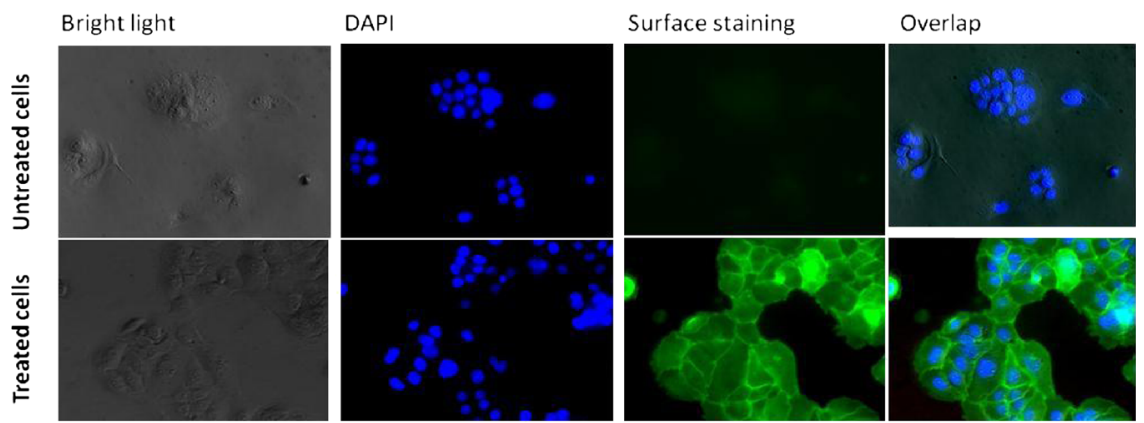

(B)

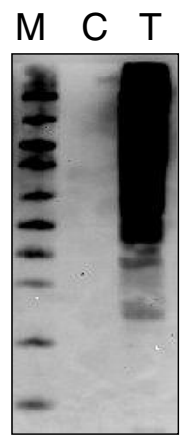

(C)

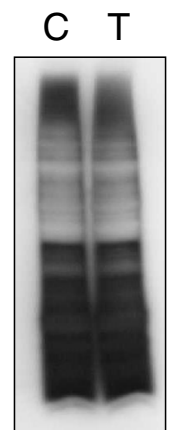

Figure 2 Assessment of metabolic incorporation of azido-modified sialic acids into cellular glycans. (A) Cell surface labeling of SW1990 cells incubated with 1,3,4-O-Bu $\mathrm{ManNAz}$ for two days. Labeling of the resulting surface azido-glycoconjugates, indicated by green fluorescence, was achieved by click chemistry with an Alexa488 fluorophore and the cells were counterstained with DAPI (blue fluorescence) as a nucleus marker. (B) Proteins were harvested from untreated control (denoted by " $\mathrm{C}$ ") and treated (denoted by "T") SW1990 cells and the specific biotinylation of azide-modified proteins was assessed by Western blots (" $\mathrm{M}^{\prime \prime}$ denotes molecular weight markers). (C) Silver staining of PAGE-separated proteins from control (denoted " $\mathrm{C}^{\prime}$ ) or treated (denoted "T") SW1990 cells verified that similar amounts of protein were analyzed in both cases.

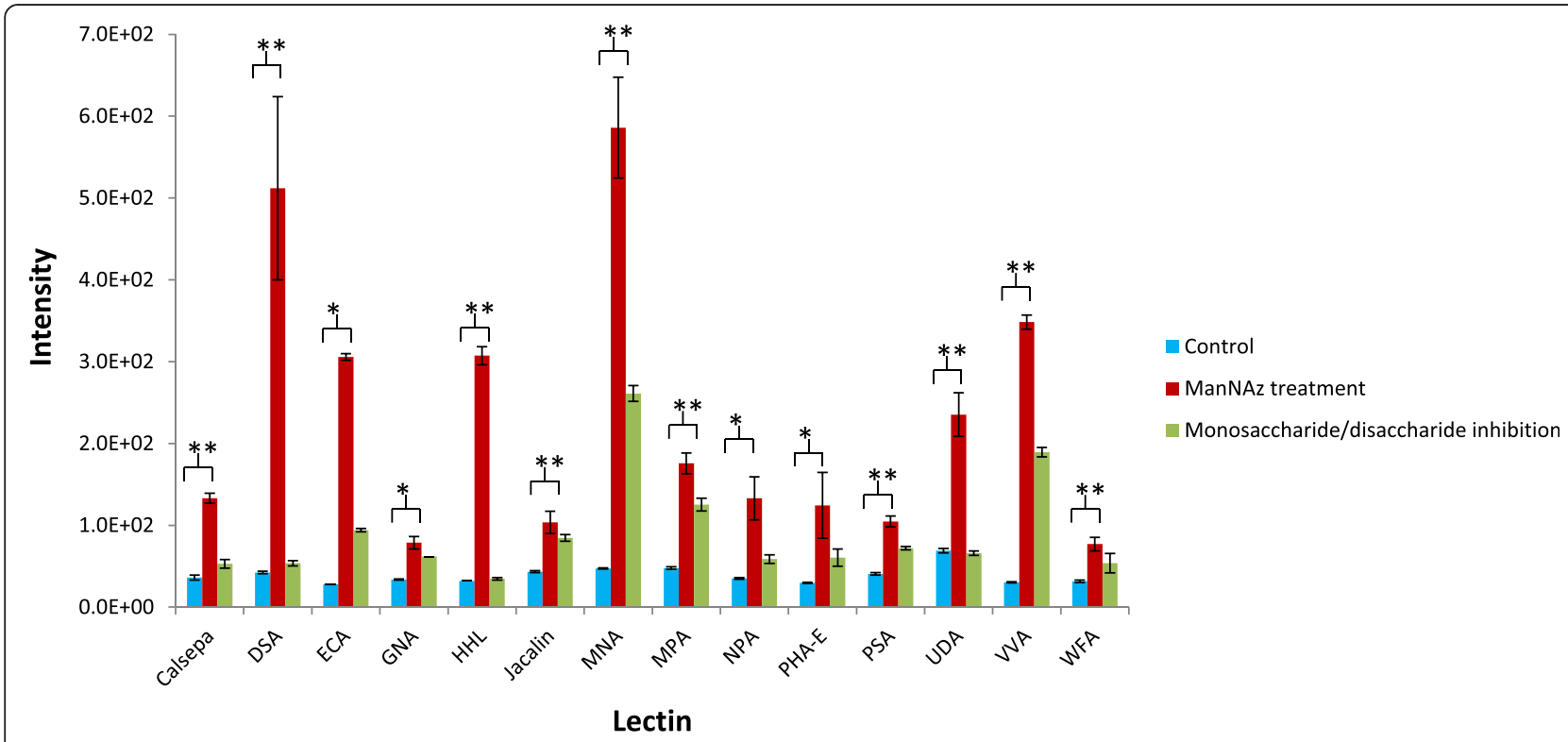

Figure 3 Glycan analysis of azido-modified glycoproteins. Glycan profiles of azido-modified glycoproteins analyzed by lectin microarray. Fourteen lectins showed significant binding to treated cells compared to control cells ( ${ }^{*} p<0.05$ and ${ }^{* *} p<0.01$ ). The lectin binding signals were inhibited by the appropriate mono- and disaccharides. 
a significant difference of $p<0.01$ above the background while binding to four additional lectins showed lower intensity that was nonetheless significantly above background levels $(p<0.05)$. Binding to the lectins was specifically inhibited by a monosaccharide or a disaccharide that competes the binding to the same lectin, which confirmed the binding signal was specific (Figure 3). Lectins with significant binding signal were DSA, PHA-E, PSA, WFA, Jacalin, MPA, ECA, UDA, GNA, HHL, NPA, Calsepa, MNA-G, and VVA (Figure 3 and Table 1). Based on the glycan-binding specificity of these lectins, among the $N$-type glycans, the azide-labeled sialoglycoproteins contained $\alpha-1-6$ core fucoses (PSA), Gal $\beta 1-4$ GlcNAc structures (DSA and PHAE), and high mannose (NPA, GNA, and HHL) (Table 1 and Additional file 1: Table S1). For O-type glycans, the glycan structure contained Gal $\beta 1$ 3GalNAc (Jacalin and MPA) and GalNAc $\beta 1-4$ GlcNAc (WFA) (Table 1 and Additional file 1: Table S1). The most intensive binding signal was detected in DSA and NPA (Figure 3).

\section{Identification and characterization of azide-labeled sialoglycoproteins by LC-MS/MS}

By using the strategy outlined in Figure 1, Steps 6 to 9, 75 unique formerly azido-sialic acid modified glycopeptides were identified in samples obtained from SW1990 cells treated with $1,3,4-\mathrm{O}-\mathrm{Bu}_{3} \mathrm{ManNAz}$ analog, representing 55 different glycoproteins (Table 2 and Additional file 2: Table S2). The MS/MS annotation of all the identified peptides is provided in Additional file 3: Table S3. No glycoproteins were identified from cells that were not treated with $1,3,4-\mathrm{O}-\mathrm{Bu}_{3} \mathrm{ManNAc}$, indicating the specific enrichment and release of azido-sialic acid containing sialoglycoproteins. Approximately $17 \%$ of the proteins were identified by two or more unique glycopeptides, e.g., galectin-3-binding protein (GAL3BP) was identified by 5 glycopeptides, and lysosome-associated membrane glycoprotein 1 (LAMP1) identified by 4 glycopeptides.

\section{Analysis of metabolically labeled sialoglycoproteins labeled with azido-sialoglycans}

To gain insight into the biological activities of the glycoproteins that had been isolated and identified from the 1,3,4-O-Bu $\mathrm{ManNAz}_{3}$ treated SW1990 cells, GO analysis was performed [38]. The analysis of cellular component shows that the majority of identified proteins (83\%) are extracellular proteins, including cell membrane proteins and secreted proteins. The analysis of molecular function shows that these glycoproteins play important roles in cell signal transduction and cell-cell interaction including catalytic activity (32.7\%), receptor activity (29.1\%), binding (25.5\%), enzyme regulator activity (5.5\%), transporter activity (5.5\%), and ion channel activity (1.8\%). The analysis of biological process again reveals a broad range of activities for the identified proteins including cellular process (15.0\%), metabolic process (14.4\%), cell communication (12.4\%), immune system process $(11.8 \%)$, cell adhesion (11.1\%), response to stimulus (8.5\%), and developmental process $(6.5 \%)$. Investigation of the identified sialoglycoproteins using previous reported glycoproteins showed that among the 55 identified sialoglycoproteins labeled by sialic acid analog, 42 proteins have documented correlation with cancer (Additional file 2: Table S2), including the $4 \mathrm{~F} 2$ cell-surface antigen, CD 109 antigen, integrin $\beta 1$, and carboxypeptidase D [39-42]. In addition, fifteen of the proteins have been specifically linked to pancreatic cancer

Table 1 Lectins showed specific binding signal to azide-modified glycoproteins

\begin{tabular}{|c|c|c|c|}
\hline Lectin & Full name & Specificity* & $p$ value \\
\hline DSA & Datura stramonium & $(\mathrm{GlcNAc} \beta 1-4) n$, Gal $\beta 1-4 \mathrm{GlcNAc}$ & $<0.01$ \\
\hline PHA-E & Phaseolus vulgaris $E$ & Complex-type N-glycans with outer Gal and bisecting GlcNAc & $<0.05$ \\
\hline PSA & Pisum sativum & Fuca1-6GlcNAc, a-D-Glc, a-D-Man & $<0.01$ \\
\hline WFA & Wisteria floribunda & GalNAcß1-4GlcNAc, Galß1-3(-6)GalNAc & $<0.01$ \\
\hline Jacalin & Artocarpas integliforia & Galß1-3GalNAc, GalNAc & $<0.01$ \\
\hline MPA & Maclura pomifera & Galß1-3GalNAc, GalNAc & $<0.01$ \\
\hline ECA & Erythrina cristagalli & Galß1-4GlcNAc (Terminal) & $<0.05$ \\
\hline UDA & Urtica dioica & GlcNAcß1-4GlcNAc, Mixture of Man5 to Man9 & $<0.01$ \\
\hline GNA & Galanthus nivalis & High-Man, Mana1-3Man & $<0.05$ \\
\hline $\mathrm{HHL}$ & Hippeastrum Hybrid & High-Man, Mana1-3Man, Mana1-6Man & $<0.01$ \\
\hline NPA & Narcissus pseudonarcissus & High-Man, Mana1-6Man & $<0.05$ \\
\hline Calsepa & Calystegia sepium & Man, maltose & $<0.01$ \\
\hline MNA-G & Morus nigra $G$ & Man & $<0.01$ \\
\hline WA & Vicia villosa & a-Linked terminal GalNAc, GalNAca1-3Gal & $<0.01$ \\
\hline
\end{tabular}

*Lectin frontier Database; http://riodb.ibase.aist.go.jp/rcmg/glycodb/LectinSearch and references [36,37]. 
Table 2 Identification of azido-sialic acid modified $\mathbf{N}$-linked sialoglycoproteins

\begin{tabular}{|c|c|c|}
\hline Protein group accessions & Protein name & Gene symbol \\
\hline 167466198 & Intercellular adhesion molecule 1 & ICAM1 \\
\hline 296010912 & Tissue factor & CD142 \\
\hline 7669492 & Glyceraldehyde-3-phosphate dehydrogenase & GAPD \\
\hline 4503143 & Cathepsin D & CTSD \\
\hline 4505467 & 5'-nucleotidase isoform 1 & NT5E \\
\hline 4507483 & Thrombomodulin & THBD \\
\hline 9845238 & UDP-GIcNAc: $\beta$ Gal $\beta-1,3-N$-acetylglucosaminyltransferase 2 & B3GNT2 \\
\hline 223468595 & Integrin a-V & Itgav \\
\hline 20150648 & Chain A,Human Dipeptidyl Peptidase I (Cathepsin C) & CTSC \\
\hline 19743823 & Integrin $\beta-1$ & |tgb1 \\
\hline 29550838 & Golgi membrane protein 1 & GOLM1 \\
\hline 53829379 & Urokinase plasminogen activator surface receptor & PLAUR \\
\hline 94962177 & Activated leukocyte cell adhesion molecule variant 1 & Alcam \\
\hline 38327634 & ATP-dependent RNA helicase DDX18 & DDX18 \\
\hline 51247095 & Chain T, Tissue Factor-Factor Viia Complex & \\
\hline 116534898 & Desmoglein-2 & DSG2 \\
\hline 4505061 & Cation-dependent mannose-6-phosphate receptor & M6PR \\
\hline 22202611 & Carboxypeptidase D & cpd \\
\hline 85544358 & Chain C, A Minimal Gas6-Axl Complex & \\
\hline 227430301 & CD109 antigen & CD109 \\
\hline 4507509 & Metalloproteinase inhibitor 1 & TIMP1 \\
\hline 189458817 & Transferrin receptor protein 1 & TFRC \\
\hline 4959370 & Radical fringe & RFNG \\
\hline 91199546 & CD63 antigen & CD63 \\
\hline 5031863 & Galectin-3-binding protein & LGALS3BP \\
\hline 6014587 & Mesothelin/megakaryocyte potentiating factor & MSLN \\
\hline 10863927 & Peptidyl-prolyl cis-trans isomerase A & PPIAL3 \\
\hline 149363636 & Plexin-B2 & plxnb2 \\
\hline 157266292 & Intestinal-type alkaline phosphatase & ALPI \\
\hline 294660768 & MHC class I polypeptide-related sequence A (MICA*00801) & MICA \\
\hline 27754771 & Protocadherin-1 & $\mathrm{PCDH} 1$ \\
\hline 222831610 & Choline transporter-like protein 2 & SLC44A2 \\
\hline 47419930 & Chondroitin sulfate proteoglycan 4 & cspg 4 \\
\hline 32967311 & Ephrin type-A receptor 2 & EPHA2 \\
\hline 4758950 & Peptidyl-prolyl cis-trans isomerase B & ppiB \\
\hline 112380628 & Lysosome-associated membrane glycoprotein 1 & lamp1 \\
\hline 4504957 & Lysosome-associated membrane glycoprotein 2 & lamp2 \\
\hline 38569398 & Integrin a-10 & ITGA10 \\
\hline 4506113 & Major prion protein & PRNP \\
\hline 21536337 & Myelin protein zero-like protein 2 & MPZL2 \\
\hline 187828910 & CD59 glycoprotein & CD59 \\
\hline 10092665 & Sushi domain-containing protein 2 & Susd2 \\
\hline 68163411 & CD166 antigen & Alcam \\
\hline 190194386 & Transmembrane 9 superfamily member 3 & TM9SF3 \\
\hline
\end{tabular}


Table 2 Identification of azido-sialic acid modified $\mathbf{N}$-linked sialoglycoproteins (Continued)

\begin{tabular}{ll}
\hline 180225 & Carcinoembryonic antigen \\
270483821 & Tetraspanin-3 \\
7657373 & Tetraspanin-13 \\
61744483 & 4F2 cell-surface antigen heavy chain \\
24308201 & Adipocyte plasma membrane-associated protein \\
5542165 & Chain A, Human Platelet Profilin Complexed With An L-Pro10- lodotyrosine Peptide \\
5453832 & Hypoxia up-regulated protein 1 \\
310125063 & HLA class I histocompatibility antigen, A-43 a chain-li \\
148728160 & Receptor-type tyrosine-protein phosphatase eta \\
166235138 & Seizure 6-like protein 2 isoform 3 \\
166235140 & Seizure 6-like protein 2 isoform 4 \\
\hline
\end{tabular}

(Additional file 2: Table S2), including cathepsin D, glyceraldehyde-3-phosphate dehydrogenase, intercellular adhesion molecule 1, mesothelin, and tissue factor [43-47].

\section{Implications of cancer-specific sialoglycoproteins} identified from metabolically glycoengineered cells

To test whether the glycoproteins identified from our metabolic oligosaccharide engineering approach have potential clinical significance (e.g., as biomarkers), immunohistological analysis was performed on cancerous and non-cancerous pancreatic tissue. Lysosome-associated membrane glycoprotein 1(LAMP1) and hypoxia upregulated protein 1 (ORP 150) were chosen for analysis in this regard because they previously have not been associated with pancreatic cancer. LAMP1 is a glycoprotein usually expressed in endosomes and lysosomal substructures of cells with less expression on the plasma membrane $[48,49]$. Elevated expression of LAMP1 at the cell surface has been reported in metastatic tumor cells although no link with pancreatic cancer has been reported [50]. Similarly, no connection with pancreatic cancer yet has been reported for ORP 150, a protein that plays a role in protein folding and secretion in the ER and hypoxia-induced cellular perturbation and is up-regulated in certain tumors, especially in breast cancer [51-55].

The protein expression of LAMP1 and ORP150 was investigated by immunohistochemistry in eight pairs of pancreatic cancer and matched non-tumorous pancreas tissue sections. All pathological materials and the IHC results were reviewed and scored by an American Pathology Boards certified pathologist. Figure 4A showed that LAMP1 was over-expressed in tumor cells but not in the paired nontumorous pancreas ductal cells. Seven of eight pancreatic cancer cases were stained strongly (score $\geq+2$ ) while no staining was detected in matched nontumorous pancreas tissues. A similar phenomenon was observed for ORP150. The expression of ORP150 in cancer cells was significantly increased as compared to adjacent tumor-matched normal pancreas ductal cells
(Figure 4B). Numbers of no/weak staining and moderate/ strong staining was compared between tumor cells and matched nontumorous pancreas ductal cells. Both LAMP1 and ORP150 showed over-expressions in pancreatic tumor cells $(P$-value $=0.0014)$.

\section{Discussion}

Glycosylation is a complex co-/post-translational modification of proteins that mediates a variety of cell surface recognition and binding events, participates in many facets of the vertebrate immune response and contribute to the progression of many diseases including the focus of this study, which is cancer [56]. Glycans, however, have remained relatively poorly understood because of challenges in identifying glycoproteins, determining the occupancy status and precise oligosaccharide structures located at specific glycosylation sites due largely to the complexity of the glycosylation process [56]. In this study, we used metabolic oligosaccharide engineering methods to identify the sialoglycoproteins modified by azido-sugar in metabolically engineered pancreatic cancer cells when the cells were over-supplied with sialic acid substrate analog.

Pancreatic cancer is the fourth leading cause of death by cancer in Europe and in the US [57]. Among the common malignancies, pancreatic cancer has the highest mortalities with a 5-year survival rate of less than 5\% [58,59]. Poor prognosis of pancreatic cancer is due to the aggressive nature of this disease, rapid metastasis, and late diagnosis of the malignancy [60]. Thus, identification of biomarkers as targets to diagnose and treat pancreatic cancer is urgently needed, which provides the impetus for our efforts to discover pancreatic cancer-associated sialoglycoproteins in the current report.

Because the heterogeneity of protein glycosylation is evidenced by a number of potential glycosylation sites on individual glycoprotein, different glycosylation sites may be modified by different carbohydrates, of which abundance may also differ from the protein expression level 


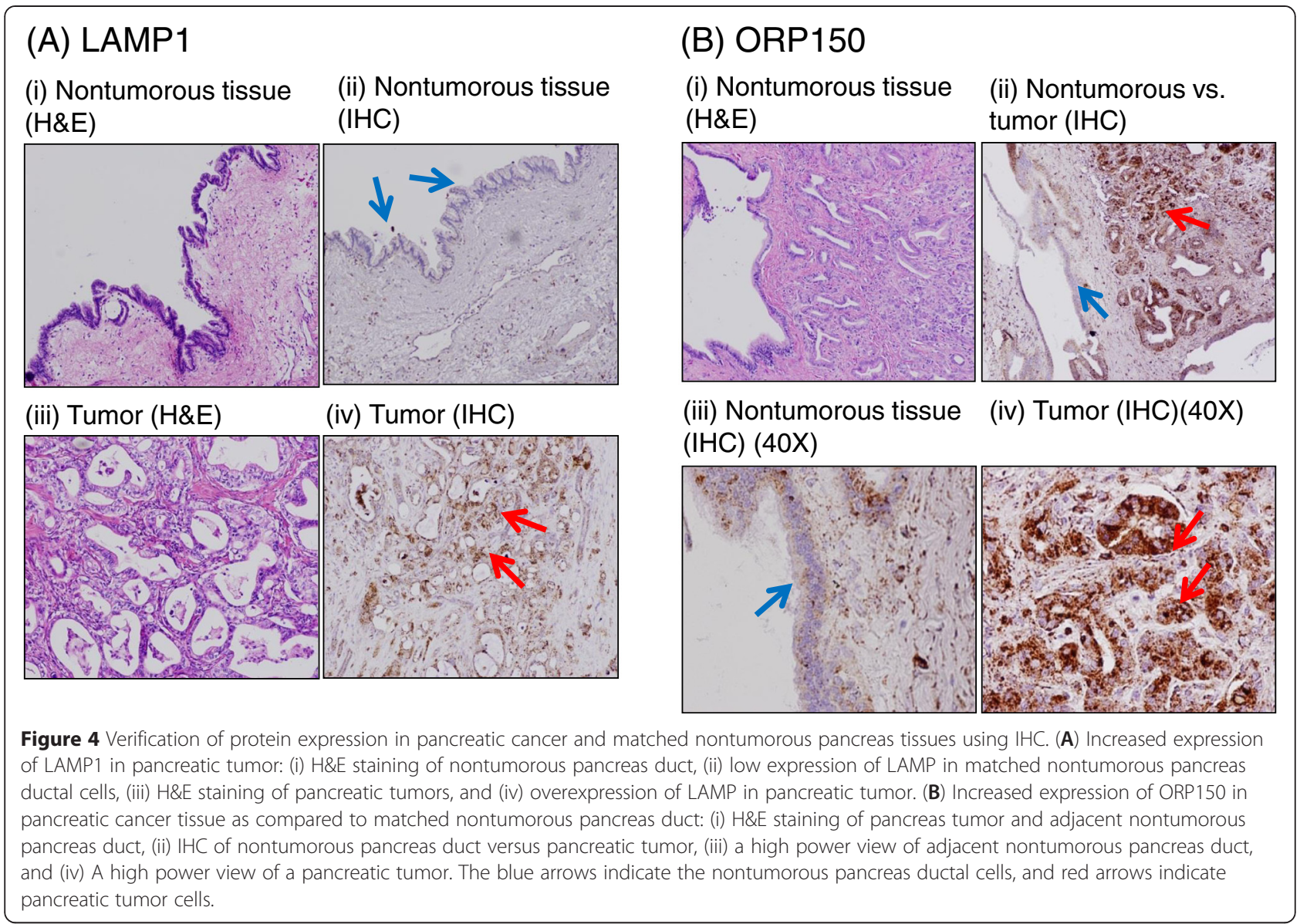

[61]. Compared to a previous strategy that used metabolic engineering to isolate sialoglycoproteins and associated proteins in the protein mixture [29], the major advantage of the approach used in this study is the capture of azido labeled sialoglycopeptides and enzymatic release of $N$ linked sialoglycopeptides for mass spectrometry identification of sialoglycosylation sites. The procedure allows the identification of not only the isolated proteins, but also the specific $N$-glycosylation sites that may contribute to more sensitive and specific identification of sialoglycoproteins [29]. Another advantage of our approach compared to previous reports is the use of synthetic azido-derivatized ManNAc analog 1,3,4-O-Bu $\mathrm{Bu}_{3} \mathrm{ManNAz}$, which is capable of supporting higher metabolic flux through the sialic acid biosynthetic pathway at low concentrations compared to $\mathrm{Ac}_{4}$ ManNAz $[29,34]$.

Seventy-five unique formerly azido-sialic acid modified glycopeptides were identified from 55 glycoproteins using 1,3,4-O- $\mathrm{Bu}_{3} \mathrm{ManNAz}$ analog treatment (Table 2 and Additional file 2: Table $\mathrm{S} 2$ ). The number of proteins we identified was relatively low compared to whole glycoproteome datasets, in which hundreds or even thousands of glycoproteins are expected to be expressed in a particular cell type. However we emphasize that we deliberately did not seek to maximize detection of all possible sialoglycans in the present study but rather conducted our experiments in a way that would be most conducive to the identification of cancer-associated biomarkers. In particular, we first limited the labeling period to two days even though evidence suggests that non-natural sialic acids can accumulate in tissue over a 6-week period upon repeated daily dosing [62]; we consider it to be unlikely that a diagnostic test could be conducted in a clinicallyrelevant manner over this extended period (instead we used a 2-day period in which a single bolus of analog has been shown to resist esterase degradation [63] and further, maximize cellular labeling without the need for added doses or replenishment). Second, the number of cells involved in each experiment $(<10 \mathrm{e} 7)$ coupled with the subfemtomolar mass spec detection sensitivity limit implies that only relatively abundant markers (e.g., glycoproteins expressed in the hundreds to thousands of copies per cell) will be detected by our methods; again, this feature is desired to ensure that our methods will have clinical relevance where sample size is likely to be limiting. Finally, as described in our previous publication [9], we use stringent 
statistical cutoffs to avoid false positives. Together, these factors - by design - limit the number of glycoproteins that are identified by our method and ensure that the glycoproteins we identify will be sufficiently abundant and metabolically labeled with sufficient kinetics to be viable cancer biomarkers.

Another matter of interest is the exact glycan structures that the non-natural azido-modified sialic acids label in cancer cells. In our previous experiments using 1,3,4-O-Bu ${ }_{3} \mathrm{ManNAc}$, which increased levels of natural sialic acid by $\sim 75 \%$ in treated cells [9], the levels of sialylation were sufficiently higher in the treated cells to allow characterization of the new glycoforms by using mass spectrometry-based methods [64]. By comparison, treatment of cells with non-natural analogs tends to result in the replacement of natural sialic acids with their non-natural counterparts rather than an overall increase in sialylation (for perspective on this issue, consult references [65-68]. In general, even though several million non-natural sialic acids can be incorporated on the surface of metabolically oligosaccharide engineered cancer cells [69], the number of these moieties constitute only a small percentage of the hundreds of millions of sialic acids typically found on each human cell [69]. Consequently, rigorous detection of non-natural sialic acids by unambiguous techniques such as mass spectrometry because of their low abundance remains extremely challenging and alternative approaches such as lectin binding assays have provided some insight.

Lectins have long been used for purification and detection of glycans in many studies [70]; many lectins have overlapping affinity while some others have unique specificity, allowing for the efficient detection of differential expression of glycan structure. In the current studies the use of a previously-reported lectin array [35] showed significant binding signal from fourteen lectins $(p<0.05)$, indicating $\alpha-1-6$ core fucoses (PSA), Gal $31-4$ GlcNAc structures (DSA and PHAE), high mannose (NPA, GNA, and HHL) among the $N$-type glycans, and Gal $\beta 1$ 3GalNAc (Jacalin and MPA) and GalNAc $\beta 1-4$ GlcNAc (WFA) for $O$-type glycans (Figure 3 and Table 1). This data provided rudimentary insight into the glycan structures compatible with azido-sialic acid incorporation but the most interesting aspect of these experiments was the lack of. lectin binding to native sialic acid despite the presence of the appropriate lectins (e.g., SNA) on the array and the plentiful display of sialic acids on SW1990 cells demonstrated in our previous experiments $[9,64]$. Possible explanations for this outcome include that 1) azido-sialic acid was not well recognized by, or had weak binding, to sialic acid specific lectins, such as SNA or that 2) the proteins modified by native sialic acid are not simultaneously modified by azido-sialic acid.
As a final part of this project, we return to the initial motivation of this study, which was to use metabolic oligosaccharide engineering methodology to identify cancerassociated biomarkers in pancreatic cancer. This task is complicated by not having a high quality "normal" pancreatic line that readily grows in cell culture conditions for comparison. Therefore, one way we attempted to address this issue was to cross-reference the list of the 55 proteins we identified against cancer (in general) and pancreatic cancer (more specifically) and found that 42 proteins have been reported to be associated with cancer in general and 15 have been specifically linked to pancreatic cancer (Additional file 2: Table S2). Although encouraging, the significance of this type of analysis is open to interpretation considering that many of the proteins are linked to cancer by as few as one reference, and further, it is not unexpected to detect cancer-related proteins by analyzing cancer cells. Therefore, we tried an alternative approach that we consider to be more stringent by analyzing clinical samples for two proteins (LAMP1 and ORP150) not previously associated with pancreatic cancer and found preferential expression in tumors compared to normal tissue in both cases (Figure 4). Although far from definitive (for example, comprehensive analysis of all the identified glycoproteins is beyond the scope of the current investigation), this early result strongly supports our metabolic oligosaccharide engineering approach as a viable strategy for the identification of new cancer biomarkers.

In conclusion, this paper describes a strategy to analyze metabolically active sialoglycoproteins found in pancreatic cancer cells and shows that robust labeling of several proteins already associated with tumors occurs. Even more significantly, the positive evaluation of two proteins not previously associated with pancreatic cancer in clinical samples suggests that this method may be a powerful technique for biomarker discovery. In summary, the results showed that metabolic oligosaccharide engineering had broader implications by the discovery of sialoglycoproteins in cancer cells that hold potential for the diagnosis of cancer or as cell surface therapeutic targets [71].

\section{Materials and methods}

\section{Cell culture}

The pancreatic cell line, SW1990, was purchased from the American Type Culture Collection (ATCC; Manasses, VA). Cells were grown in 1:1 DMEM medium supplemented with $10 \%(\mathrm{v} / \mathrm{v})$ FBS and $1 \%(\mathrm{v} / \mathrm{v})$ of a solution of 10,000 units penicillin and $10 \mathrm{mg}$ streptomycin $/ \mathrm{mL}$, at $37^{\circ} \mathrm{C}$ and $5 \% \mathrm{CO}_{2}$.

\section{Metabolic labeling of cells and cell surface azide visualization via fluorescence microscopy}

Pancreatic cells were seeded on 6-well TC plates and synthetic 1,3,4-O- $\mathrm{Bu}_{3} \mathrm{ManNAz}$ analog [34] from a $50 \mathrm{mM}$ 
stock solution was added to each well prior seeding the cells to provide a final concentration of $50 \mu \mathrm{M}$. Cells were incubated for two days with or without the analog. Prior to visualization, the cells were washed with PBS and fixed with $3.7 \%$ formaldehyde for $20 \mathrm{~min}$ at room temperature. Labeling was done by adding $200 \mu \mathrm{L}$ of a freshly prepared Click Reaction Mixture to each well followed by incubation in the dark for $45 \mathrm{~min}$ as described previously [34]. The cells were then washed 3-5 times with PBS containing 5\% BSA. Images were taken using a Zeiss Observer microscope with a 40X objective lens (Zeiss, Inc., Melville, NY). Fluorescence pictures of Alexa488 (surface sialoglycoconjugates), and DAPI (nucleus) labeled cells were recorded and overlay images were generated with the Zeiss Imaging System.

Biotinylation of azido-sialic acid containing glycoproteins To selectively biotinylate azido-sialic acid modified glycoproteins, the modified Staudinger ligation reaction reported by Saxon and coworkers using Biotin-PEG P $_{3}$-Phosphine was performed on proteins extracted from cell lysates [15]. The protein concentration was adjusted to $2.5 \mathrm{mg} / \mathrm{mL}$ in PBS (pH 7.2), Biotin- $\mathrm{PEG}_{3}$-Phosphine was added to a final concentration of $200 \mu \mathrm{M}$. The sample was incubated overnight at room temperature. The biotinylation efficiency and specificity was examined by Western blot using HRP-streptavidin at 1:5000. The non-reacted Biotin$\mathrm{PEG}_{3}$-Phosphine was removed through protein precipitation. The proteins were mixed with an 8-fold volume of pre-chilled $\left(-20^{\circ} \mathrm{C}\right)$ acetone by vortexing and then incubated at $-20^{\circ} \mathrm{C}$ for $60 \mathrm{~min}$. The samples were centrifuged at $13,000 \times \mathrm{g}$ for $10 \mathrm{~min}$. The supernatant was removed and disposed without disturbing the protein pellet. This washing and precipitation process was repeated and residual acetone was allowed to evaporate from the uncapped tube at room temperature for $30 \mathrm{~min}$.

\section{Affinity purification of biotinylated proteins}

A $50 \%$ slurry of monomeric avidin agarose $(1.4 \mathrm{~mL})$ resin was sequentially washed with $3 \mathrm{~mL}$ PBS, $3 \mathrm{~mL}$ of $2 \mathrm{mM}$ D-biotin in PBS, $3 \mathrm{~mL}$ of $0.1 \mathrm{M}$ glycine ( $\mathrm{pH} 2.8$ ), and $3 \mathrm{~mL}$ PBS. Protein $(500 \mu \mathrm{g}$ per sample, isolated from treated or untreated cells as described above) was added to the pre-conditioned monomeric avidin agarose resin and incubated at room temperature for $1 \mathrm{~h}$, followed by five washes with $6 \mathrm{~mL}$ PBS. The biotinylated azido-modified glycoproteins were eluted with $0.7 \mathrm{~mL}$ of $2 \mathrm{mM}$ D-biotin in PBS for $10 \mathrm{~min}$ followed by a second elution step of $0.7 \mathrm{~mL}$ of $0.1 \mathrm{M}$ glycine (pH 2.8) for $10 \mathrm{~min}$.

\section{Characterized sialic acid glycans using lectin microarrays}

A lectin microarray comprised of 38 lectins as described in our previous study was used to profile the glycan structures of the proteins biotinylated via Staudinger ligation [35]. Each lectin was prepared in three concentrations $(1,0.5$, and $0.25 \mathrm{mg} / \mathrm{mL})$ on the chip. The slides were blocked with $50 \mathrm{mM}$ ethanolamine in $40 \mathrm{mM}$ sodium borate for $1 \mathrm{~h}$ followed by three subsequent washes with PBS- $0.1 \%$ Tween. The biotinylated proteins $(100 \mu \mathrm{L})$ were applied to each lectin microarray. Inhibitory sugar solutions (e.g. $100 \mu \mathrm{L}$ of $50 \mathrm{mM}$ lactose, $50 \mathrm{mM}$ galactose, $50 \mathrm{mM}$ mannose, or $50 \mathrm{mM}$ acetic acid) were mixed with samples, and then were applied to the control wells. After an overnight incubation at room temperature, each well was washed with $100 \mu \mathrm{L}$ of PBS- $0.1 \%$ Tween 20 with 5 min shaking. Rabbit IgG $(20 \mu \mathrm{g})$ was added to each array followed by a $30 \mathrm{~min}$ incubation to mask any residual lectin sites. The slides were washed three times with PBS-0.1\% Tween 20. Afterwards, $100 \mu \mathrm{L}$ of streptavidin conjugated to Dylight 549 (diluted 500-fold in PBS$0.1 \%$ Tween) was added to each well and incubated for $30 \mathrm{~min}$ in the dark before the array was washed, dried, and scanned with a GenePix 4100B scanner (Sunnyvale, CA).

\section{Identification of formerly azide-sialic acid modified glycopeptides}

After biotinylation and removal of the non-reacted Biotin$\mathrm{PEG}_{3}$-Phosphine using protein precipitation, $1 \mathrm{mg}$ of protein was denatured and reduced in $8 \mathrm{M}$ urea, $10 \mathrm{mM}$ TCEP, and $1 \%$ SDS in $200 \mathrm{mM}$ Tris buffer at $37^{\circ} \mathrm{C}$ for $2 \mathrm{~h}$ [72]. Iodoacetamide (10 $\mathrm{mM}$ final concentration) was added to the sample and incubated for $30 \mathrm{~min}$ at RT in the dark. The solution was diluted 8-fold with $100 \mathrm{mM}$ $\mathrm{KH}_{2} \mathrm{PO} 4$ ( $\mathrm{pH}$ 8.0). The proteins were digested by trypsin (Promega, 1:50, w/w) at $37^{\circ} \mathrm{C}$ overnight with shaking. After digestion, the samples were centrifuged at $16,110 \times$ $\mathrm{g}$ for $5 \mathrm{~min}$ to remove particulate matter. The peptides were cleaned by using a Sep-Pak C18 Vac cartridge (Waters) and resuspended in PBS. Six hundred microliters of $50 \%$ slurry of streptavidin agarose resin was washed with $1 \mathrm{~mL}$ PBS (pH 7.2) three times. Protease inhibitor (Pierce) was added to the peptides at a 1:1000 dilution prior to mixing peptides with pre-washed streptavidin agarose. The peptides were allowed to bind to streptavidin agarose at room temperature for $15 \mathrm{~min}$. The unbound peptides were removed by centrifugation. The streptavidin agarose was washed with $1 \mathrm{~mL}$ PBS 6 times, then resuspended in $250 \mu \mathrm{L}$ G7 buffer (New England Biolabs) and peptides containing $\mathrm{N}$-linked glycosylation sites with metabolic labeled sialoglyans were released by PNGase F (New England Biolabs) [73-75]. The peptides were analyzed using a LTQ-Orbitrap velos (ThermoFisher, Waltham, MA) coupled with a $15 \mathrm{~cm} \times 75 \mu \mathrm{m} \mathrm{C18}$ column ( $5 \mu \mathrm{m}$ particles with 100 angstrom pore size). The spectra were analyzed as described in our previous report [76]. Briefly, a nanoAquity UPLC at $300 \mathrm{~nL} / \mathrm{min}$ with a 90 min linear acetonitrile gradient (from 5-32\% B over 
90 min; $\mathrm{A}=0.1 \%$ formic acid in water, $\mathrm{B}=0.1 \%$ formic acid in acetonitrile) was used. A top 10 data dependent MS/MS with exclusion for $20 \mathrm{~s}$ was set.

MS/MS spectra were searched with MASCOT (version 2.2.0) using Proteome Discoverer (version 1.0) (Thermo Fisher) against human subdatabase of NCBI Reference Sequence (RefSeq) (version 40, released at April 16, 2010) containing 29,704 sequences. For this database search, the precursor mass tolerance and fragment mass tolerance were set at $15 \mathrm{ppm}$ and $0.05 \mathrm{Da}$ respectively. Trypsin was specified as the protease. The fixed modification was set as carbamidomethylation $(C)$, and other database-searching parameters were set as flexible modification as follows: deamidation (NQ) and oxidation (M). Semi-tryptic end and one missed cleavage site was allowed. The False Discovery Rate was set at 0.01 to eliminate lowprobability protein identifications.

Identified proteins were classified according to their main biological processes, their molecular functions and their cellular component using "Blast2Go" software (www.blast2go.com) [38].

\section{Immunohistochemistry (IHC)}

Formalin-fixed and paraffin-embedded tumor tissue was obtained from the Department of Pathology at Johns Hopkins Hospital, and was cut into 4-micron sections. Slides were de-paraffined and rehydrated before IHC study. Dako LSAB+ System-AP kit (Carpinteria, CA) used for IHC study according to the manufacturer's instructions. Antigen-retrieval using $10 \mathrm{mM}$ sodium citrate (pH 6.0) at $100^{\circ} \mathrm{C}$ for 20 min was performed prior to apply mouse monoclonal anti- human LAMP1 antibody at 1:150 dilution. The mouse monoclonal anti- human ORP150 antibody was applied to slides at 1:200 dilution without antigen retrieval. The primary antibodies were incubated overnight at $4^{\circ} \mathrm{C}$. All pathological materials and IHC results were reviewed and evaluated by an American Pathology Boards certified pathologist, Dr. Qing Kay Li. The cytoplasmic immuno-staining pattern and immunoreactive intensity were scored semi-quantitatively using a 4 tier system: no staining $(0)$, weak $(1+,<10 \%$ positivity), moderate $(2+, 10-50 \%$ positivity), and strong $(3+,>50 \%$ positivity). The IHC staining pattern was correlated with tumors' pathological stages and the differentiations. Patients' clinical information was also correlated. Fisher's exact test was used to determine the statistical significance of IHC staining in pancreas tumor and paired normal pancreas tissues.

\section{Additional files}

Additional file 1: Table S1. Information of 38 lectins used in lectin microarray.
Additional file 2: Table S2. Identification of N-glycosite-containing sialoglycopeptides using LC-MS/MS.

Additional file 3: Table S3. MS/MS annotation for the identified sialoglycopeptides.

Competing interests

The authors declare that they have no competing interests.

\section{Authors' contributions}

YT participated in the study design and carried out the sialoglycopeptide/ sialoglycoprotein isolation, sample preparation for MS analysis, and drafted the manuscript. RA and CS carried out the cell metabolic labeling. CC and QL carried out the immunohistochemistry analysis supporting this study. DL helped with lectin microarray analysis. PS carried out bioinformatics analysis. $\mathrm{KJY}$ and $\mathrm{HZ}$ participated in the study design and manuscript preparation. All authors read and approved the final manuscript.

\section{Acknowledgements}

This work was supported by federal funds from the National Cancer Institute, National Institutes of Health, grants 2R01CA112315, U01CA152813, R01CA112314, and P01HL107153-01. We also gratefully acknowledge the support of Drs. Robert Cole and Robert O'Meally from Johns Hopkins University for their assistance in mass spectrometry analysis and data processing.

\section{Author details}

'Department of Pathology, Johns Hopkins University, 400 N. Broadway, Room 4011, Baltimore MD 21287, USA. ²Department of Biomedical Engineering and the Translational Tissue Engineering Center, Johns Hopkins University School of Medicine, Baltimore, MD, USA.

Received: 3 October 2014 Accepted: 23 March 2015

Published online: 11 April 2015

\section{References}

1. Varki A. Glycan-based interactions involving vertebrate sialic-acid-recognizing proteins. Nature. 2007:446(7139):1023-9.

2. Hakomori S. Tumor-associated carbohydrate antigens. Annu Rev Immunol. 1984;2:103-26.

3. Fukuda M. Possible roles of tumor-associated carbohydrate antigens. Cancer Res. 1996:56(10):2237-44.

4. Schauer R. Achievements and challenges of sialic acid research. Glycoconj J. 2000;17(7-9):485-99.

5. Schultz MJ, Swindall AF, Bellis SL. Regulation of the metastatic cell phenotype by sialylated glycans. Cancer Metastasis Rev. 2012;31(3-4):501-18.

6. Meany DL, Zhang Z, Sokoll LJ, Zhang H, Chan DW. Glycoproteomics for prostate cancer detection: changes in serum PSA glycosylation patterns. J Proteome Res. 2009;8(2):613-9.

7. Tian Y, Esteva FJ, Song J, Zhang H. Altered expression of sialylated glycoproteins in breast cancer using hydrazide chemistry and mass spectrometry. Mol Cell Proteomics. 2012;11(6):M111 011403.

8. Keppler OT, Hinderlich S, Langner J, Schwartz-Albiez R, Reutter W, Pawlita M. UDP-GlcNAc 2-epimerase: a regulator of cell surface sialylation. Science. 1999;284(5418):1372-6

9. Almaraz RT, Tian Y, Bhattarcharya R, Tan E, Chen SH, Dallas MR, et al. Metabolic flux increases glycoprotein sialylation: implications for cell adhesion and cancer metastasis. Mol Cell Proteomics. 2012;11(7):M112 017558.

10. Zhao J, Simeone DM, Heidt D, Anderson MA, Lubman DM. Comparative serum glycoproteomics using lectin selected sialic acid glycoproteins with mass spectrometric analysis: application to pancreatic cancer serum. J Proteome Res. 2006;5(7):1792-802.

11. Malykh YN, Schauer R, Shaw L. N-Glycolylneuraminic acid in human tumours. Biochimie. 2001;83(7):623-34

12. Bardor M, Nguyen DH, Diaz S, Varki A. Mechanism of uptake and incorporation of the non-human sialic acid N-glycolylneuraminic acid into human cells. J Biol Chem. 2005;280(6):4228-37.

13. Pan Y, Chefalo P, Nagy N, Harding C, Guo Z. Synthesis and immunological properties of $\mathrm{N}$-modified $\mathrm{GM} 3$ antigens as therapeutic cancer vaccines. J Med Chem. 2005;48(3):875-83.

14. Mahal LK, Yarema KJ, Bertozzi CR. Engineering chemical reactivity on cell surfaces through oligosaccharide biosynthesis. Science. 1997;276(5315):1125-8. 
15. Saxon E, Bertozzi CR. Cell surface engineering by a modified Staudinger reaction. Science. 2000;287(5460):2007-10.

16. Neves AA, Stockmann H, Harmston RR, Pryor HJ, Alam IS, Ireland-Zecchini $H$, et al. Imaging sialylated tumor cell glycans in vivo. FASEB J. 2011;25(8):2528-37.

17. Tian Y, Zhang H. Glycoproteomics and clinical applications. Proteomics: Clin Appl. 2010;4(2):124-32.

18. Li Y, Tao SC, Bova GS, Liu AY, Chan DW, Zhu H, et al. Detection and verification of glycosylation patterns of glycoproteins from clinical specimens using lectin microarrays and lectin-based immunosorbent assays. Anal Chem. 2011;83(22):8509-16

19. Larsen MR, Jensen SS, Jakobsen LA, Heegaard NH. Exploring the sialiome using titanium dioxide chromatography and mass spectrometry. Mol Cell Proteomics. 2007:6(10):1778-87.

20. Lewandrowski U, Zahedi RP, Moebius J, Walter U, Sickmann A. Enhanced $\mathrm{N}$-glycosylation site analysis of sialoglycopeptides by strong cation exchange prefractionation applied to platelet plasma membranes. Mol Cell Proteomics. 2007:6(11):1933-41.

21. Gundry RL, Raginski K, Tarasova Y, Tchernyshyov I, Bausch-Fluck D, Elliott ST, et al. The mouse C2C12 myoblast cell surface N-linked glycoproteome: identification, glycosite occupancy, and membrane orientation. Mol Cell Proteomics. 2009;8(11):2555-69.

22. Wollscheid B, Bausch-Fluck D, Henderson C, O'Brien R, Bibel M, Schiess R, et al. Mass-spectrometric identification and relative quantification of $\mathrm{N}$-linked cell surface glycoproteins. Nat Biotechnol. 2009;27(4):378-86.

23. Kurogochi M, Matsushista T, Amano M, Furukawa J, Shinohara Y, Aoshima M, et al. Sialic acid-focused quantitative mouse serum glycoproteomics by multiple reaction monitoring assay. Mol Cell Proteomics. 2010;9(11):2354-68.

24. Li Y, Tian Y, Rezai T, Prakash A, Lopez MF, Chan DW, et al. Simultaneous analysis of glycosylated and sialylated prostate-specific antigen revealing differential distribution of glycosylated prostate-specific antigen isoforms in prostate cancer tissues. Anal Chem. 2011;83(1):240-5.

25. Ghesquiere B, Buyl L, Demol H, Van Damme J, Staes A, Timmerman E, et al. A new approach for mapping sialylated $\mathrm{N}$-glycosites in serum proteomes. J Proteome Res. 2007;6(11):4304-12.

26. Kayser H, Zeitler R, Kannicht C, Grunow D, Nuck R, Reutter W. Biosynthesis of a nonphysiological sialic acid in different rat organs, using $\mathrm{N}$-propanoylD-hexosamines as precursors. J Biol Chem. 1992;267(24):16934-8.

27. Bond MR, Kohler JJ. Chemical methods for glycoprotein discovery. Curr Opin Chem Biol. 2007;11(1):52-8.

28. Laughlin ST, Agard NJ, Baskin JM, Carrico IS, Chang PV, Ganguli AS, et al. Metabolic labeling of glycans with azido sugars for visualization and glycoproteomics. Methods Enzymol. 2006:415:230-50.

29. Yang L, Nyalwidhe JO, Guo S, Drake RR, Semmes OJ. Targeted identification of metastasis-associated cell-surface sialoglycoproteins in prostate cancer. Mol Cell Proteomics. 2011;10(6):M110 007294.

30. Longwell SA, Dube DH. Deciphering the bacterial glycocode: recent advances in bacterial glycoproteomics. Curr Opin Chem Biol. 2013;17(1):41-8.

31. Champasa K, Longwell SA, Eldridge AM, Stemmler EA, Dube DH. Targeted identification of glycosylated proteins in the gastric pathogen Helicobacter pylon (Hp). Mol Cell Proteomics. 2013;12(9):2568-86.

32. Slade $\mathrm{PG}$, Hajivandi M, Bartel CM, Gorfien SF. Identifying the CHO secretome using mucin-type O-linked glycosylation and click-chemistry. J Proteome Res. 2012;11(12):6175-86.

33. Mathew MP, Tan E, Saeui CT, Bovonratwet P, Liu L, Bhattacharya R, et al. Metabolic glycoengineering sensitizes drug-resistant pancreatic cancer cells to tyrosine kinase inhibitors erlotinib and gefitinib. Bioorg Med Chem Lett. 2015;25(6):1223-7.

34. Almaraz RT, Aich U, Khanna HS, Tan E, Bhattacharya R, Shah S, et al. Metabolic oligosaccharide engineering with $\mathrm{N}$-acyl functionalized ManNAC analogs: cytotoxicity, metabolic flux, and glycan-display considerations. Biotechnol Bioeng. 2012;109(4):992-1006.

35. Meany DL, Hackler Jr L, Zhang H, Chan DW. Tyramide signal amplification for antibody-overlay lectin microarray: a strategy to improve the sensitivity of targeted glycan profiling. J Proteome Res. 2011;10(3):1425-31.

36. Hirabayashi J, Kuno A, Tateno H. Lectin-based structural glycomics: a practical approach to complex glycans. Electrophoresis. 2011;32(10):1118-28.

37. Fry SA, Afrough B, Lomax-Browne HJ, Timms JF, Velentzis LS, Leathem AJ. Lectin microarray profiling of metastatic breast cancers. Glycobiology. 2011;21(8):1060-70.
38. Conesa A, Gotz S, Garcia-Gomez JM, Terol J, Talon M, Robles M. Blast2GO: a universal tool for annotation, visualization and analysis in functional genomics research. Bioinformatics. 2005;21(18):3674-6

39. Hagikura M, Murakumo Y, Hasegawa M, Jijiwa M, Hagiwara S, Mii S, et al. Correlation of pathological grade and tumor stage of urothelial carcinomas with CD109 expression. Pathol Int. 2010:60(11):735-43.

40. Nawashiro H, Otani N, Shinomiya N, Fukui S, Ooigawa H, Shima K, et al. L-type amino acid transporter 1 as a potential molecular target in human astrocytic tumors. Int J Cancer. 2006;119(3):484-92.

41. Zhou G, Chiu D, Qin D, Niu L, Cai J, He L, et al. Detection and clinical significance of $\mathrm{CD} 44 \mathrm{v} 6$ and integrin-beta1 in pancreatic cancer patients using a triplex real-time RT-PCR assay. Appl Biochem Biotechnol. 2012;167(8):2257-68.

42. Galamb O, Gyorffy B, Sipos F, Spisak S, Nemeth AM, Miheller P, et al. Inflammation, adenoma and cancer: objective classification of colon biopsy specimens with gene expression signature. Dis Markers. 2008;25(1):1-16.

43. Tsume Y, Amidon GL. The feasibility of enzyme targeted activation for amino acid/dipeptide monoester prodrugs of floxuridine; cathepsin D as a potential targeted enzyme. Molecules. 2012;17(4):3672-89.

44. Menon R, Zhang Q, Zhang Y, Fermin D, Bardeesy N, DePinho RA, et al. Identification of novel alternative splice isoforms of circulating proteins in a mouse model of human pancreatic cancer. Cancer Res. 2009;69(1):300-9.

45. Roland CL, Dineen SP, Toombs JE, Carbon JG, Smith CW, Brekken RA, et al. Tumor-derived intercellular adhesion molecule-1 mediates tumor-associated leukocyte infiltration in orthotopic pancreatic xenografts. Exp Biol Med. 2010;235(2):263-70.

46. Tang Z, Qian $\mathrm{M}, \mathrm{Ho} \mathrm{M}$. The role of mesothelin in tumor progression and targeted therapy. Anticancer Agents Med Chem. 2013;13(2):276-80.

47. Gerotziafas GT, Galea V, Mbemba E, Khaterchi A, Sassi M, Baccouche H, et al. Tissue factor over-expression by human pancreatic cancer cells BXPC3 is related to higher prothrombotic potential as compared to breast cancer cells MCF7. Thromb Res. 2012;129(6):779-86.

48. Granger BL, Green SA, Gabel CA, Howe CL, Mellman I, Helenius A. Characterization and cloning of lgp110, a lysosomal membrane glycoprotein from mouse and rat cells. J Biol Chem. 1990;265(20):12036-43.

49. Holcombe RF, Baethge BA, Stewart RM, Betzing K, Hall VC, Fukuda M, et al. Cell surface expression of lysosome-associated membrane proteins (LAMPs) in scleroderma: relationship of lamp2 to disease duration, anti-Sc170 antibodies, serum interleukin-8, and soluble interleukin-2 receptor levels. Clin Immunol Immunopathol. 1993;67(1):31-9.

50. Heffernan M, Yousefi S, Dennis JW. Molecular characterization of P2B/LAMP-1, a major protein target of a metastasis-associated oligosaccharide structure. Cancer Res. 1989:49(21):6077-84.

51. Ozawa K, Kuwabara K, Tamatani M, Takatsuji K, Tsukamoto Y, Kaneda S, et al. 150-kDa oxygen-regulated protein (ORP150) suppresses hypoxia-induced apoptotic cell death. J Biol Chem. 1999;274(10):6397-404.

52. Asahi H, Koshida K, Hori O, Ogawa S, Namiki M. Immunohistochemical detection of the 150-kDa oxygen-regulated protein in bladder cancer. BJU Int. 2002;90(4):462-6.

53. Namba T, Hoshino T, Tanaka K, Tsutsumi S, Ishihara T, Mima S, et al. Up-regulation of 150-kDa oxygen-regulated protein by celecoxib in human gastric carcinoma cells. Mol Pharmacol. 2007;71(3):860-70.

54. Tsukamoto Y, Kuwabara K, Hirota S, Kawano K, Yoshikawa K, Ozawa K, et al. Expression of the 150-kd oxygen-regulated protein in human breast cancer. Lab Invest. 1998;78(6):699-706.

55. Stojadinovic A, Hooke JA, Shriver CD, Nissan A, Kovatich AJ, Kao TC, et al. HYOU1/Orp150 expression in breast cancer. Med Sci Monit. 2007:13(11):BR231-9.

56. Hanson SR, Hsu TL, Weerapana E, Kishikawa K, Simon GM, Cravatt BF, et al. Tailored glycoproteomics and glycan site mapping using saccharide-selective bioorthogonal probes. J Am Chem Soc. 2007;129(23):7266-7.

57. Niederhuber JE, Brennan MF, Menck HR. The national cancer data base report on pancreatic cancer. Cancer Lett. 1995;76(9):1671-7.

58. Wray CJ, Ahmad SA, Matthews JB, Lowy AM. Surgery for pancreatic cancer: recent controversies and current practice. Gastroenterology. 2005;128(6):1626-41.

59. Jemal A, Siegel R, Ward E, Hao Y, Xu J, Thun MJ. Cancer statistics, 2009. CA Cancer J Clin. 2009;59(4):225-49.

60. Zhao J, Patwa TH, Qiu W, Shedden K, Hinderer R, Misek DE, et al. Glycoprotein microarrays with multi-lectin detection: unique lectin binding patterns as a tool for classifying normal, chronic pancreatitis and pancreatic cancer sera. J Proteome Res. 2007;6(5):1864-74. 
61. Ozohanics O, Turiak L, Puerta A, Vekey K, Drahos L. High-performance liquid chromatography coupled to mass spectrometry methodology for analyzing site-specific N-glycosylation patterns. J Chromatogr A. 2012;1259:200-12.

62. Gagiannis D, Gossrau R, Reutter W, Zimmermann-Kordmann M, Horstkorte R. Engineering the sialic acid in organs of mice using Npropanoylmannosamine. Biochim Biophys Acta. 2007;1770(2):297-306.

63. Mathew MP, Tan E, Shah S, Bhattacharya R, Adam Meledeo M, Huang J, et al. Extracellular and intracellular esterase processing of SCFA-hexosamine analogs: implications for metabolic glycoengineering and drug delivery. Bioorg Med Chem Lett. 2012;22(22):6929-33.

64. Shah P, Yang S, Sun S, Aiyetan P, Yarema KJ, Zhang H. Mass spectrometric analysis of sialylated glycans with use of solid-phase labeling of sialic acids. Anal Chem. 2013;85(7):3606-13.

65. Kim EJ, Sampathkumar SG, Jones MB, Rhee JK, Baskaran G, Goon S, et al. Characterization of the metabolic flux and apoptotic effects of O-hydroxyl- and $\mathrm{N}$-acyl-modified N-acetylmannosamine analogs in Jurkat cells. J Biol Chem. 2004;279(18):18342-52

66. Du J, Meledeo MA, Wang Z, Khanna HS, Paruchuri VD, Yarema KJ. Metabolic glycoengineering: sialic acid and beyond. Glycobiology. 2009;19(12):1382-401.

67. Dafik L, D'Alarcao M, Kumar K. Modulation of cellular adhesion by glycoengineering. J Med Chem. 2010;53(10):4277-84.

68. Almaraz RT, Mathew MP, Tan E, Yarema KJ. Metabolic oligosaccharide engineering: implications for selectin-mediated adhesion and leukocyte extravasation. Ann Biomed Eng. 2012;40(4):806-15.

69. Yarema KJ, Bertozzi CR. Chemical approaches to glycobiology and emerging carbohydrate-based therapeutic agents. Curr Opin Chem Biol. 1998;2(1):49-61.

70. Hirabayashi J, Yamada M, Kuno A, Tateno H. Lectin microarrays: concept, principle and applications. Chem Soc Rev. 2013;42(10):4443-58.

71. Zhang H, Chan DW. Cancer biomarker discovery in plasma using a tissue-targeted proteomic approach. Cancer Epidemiol Biomarkers Prev. 2007;16(10):1915-7.

72. Sun S, Zhou JY, Yang W, Zhang H. Inhibition of protein carbamylation in urea solution using ammonium-containing buffers. Anal Biochem. 2014;446:76-81.

73. Zhang H, Li XJ, Martin DB, Aebersold R. Identification and quantification of $\mathrm{N}$-linked glycoproteins using hydrazide chemistry, stable isotope labeling and mass spectrometry. Nat Biotechnol. 2003;21(6):660-6.

74. Zhang $\mathrm{H}$, Aebersold R. Isolation of glycoproteins and identification of their N-linked glycosylation sites. Methods Mol Biol. 2006:328:177-85.

75. Tian Y, Zhou Y, Elliott S, Aebersold R, Zhang H. Solid-phase extraction of N-linked glycopeptides. Nat Protoc. 2007;2(2):334-9.

76. Tian Y, Bova GS, Zhang H. Quantitative glycoproteomic analysis of optima cutting temperature-embedded frozen tissues identifying glycoproteins associated with aggressive prostate cancer. Anal Chem. 2011;83(18):7013-9.

\section{Submit your next manuscript to BioMed Central and take full advantage of:}

- Convenient online submission

- Thorough peer review

- No space constraints or color figure charges

- Immediate publication on acceptance

- Inclusion in PubMed, CAS, Scopus and Google Scholar

- Research which is freely available for redistribution 